\title{
Transmission ion microscopy and time-of-flight spectroscopy
}

Michael Mousley ${ }^{1}$, Wolfhard Moeller ${ }^{2}$, Patrick Philipp ${ }^{1}$, Olivier Bouton ${ }^{1}$, Nico Klingner ${ }^{3}$, Eduardo Serralta $^{3}$, Gregor Hlawacek ${ }^{4}$, Tom Wirtz ${ }^{5}$ and Santhana Eswara ${ }^{1}$

${ }^{1}$ Luxembourg Institute of Science and Technology, Belvaux, Luxembourg, ${ }^{2}$ Helmholtz-Zentrum DresdenRossendorf, dresden, Germany, ${ }^{3}$ Helmholtz-Zentrum Dresden-Rossendorf, United States, ${ }^{4}$ HelmholtzZentrum Dresden-Rossendorf, Dresden, Sachsen, Germany, ${ }^{5}$ Luxembourg Institute of Science and Technology (LIST), Belvaux, Luxembourg

A Transmission Ion Microscope (TIM), the Galileo prototype, has been built at the Luxembourg Institute of Science and Technology (LIST) [1]. This is part of a new interest in the imaging properties of transmitted helium ions [2] [3] [4]. This allows the combination of both helium ions and neutrals to be detected after passing through a sample, or, if post sample deflection is used, then only the signal from the neutrals (Figure $1 \mathrm{~A}$ ). The helium ions have an energy between $10 \mathrm{keV}$ and $20 \mathrm{keV}$ and are produced in a Duoplasmatron ion source.

The prototype instrument is very flexible and uses a microchannel plate (MCP) which can be configured in multiple ways to enable analysis modalities, producing datasets of varying dimensionality. 2D images can be obtained either with a phosphor screen and a defocussed beam in direct TIM mode (analogous to transmission electron microscopy) or with an anode plate to collect the total detector signal in scanning mode providing scanning-TIM (STIM) images. By using fast blanking electronics (similar to [5]), pulses of ions can be used to add time-of-flight (TOF) information, allowing a TOF-STIM mode to collect 3D datasets $(\mathrm{x}, \mathrm{y}, \mathrm{t})$. Alternatively, a delay line detector (DLD) can be used to provide detector plane images with corresponding TOF values at each detector pixel, to collect TOF-TIM 3D datasets (x', y', t). Finally, detector imaged DLD TOF-STIM can be used, in this mode, for each beam position on the sample, the arrival time and position on the detector is recorded for each count (5D datasets, $x, y, x$ ', y', t). The prototype TIM is also equipped with a secondary electron (SE) detector providing additional SE intensity for each pixel position in STIM modes (Figure 1 B).

Example TOF datasets from materials science related samples (e.g. Au on Si) will be presented. In addition to microscopy, the effects of $20 \mathrm{keV}$ helium ion irradiation on Au-Silica core-shell nanoparticles have been evaluated. Using bright field Transmission Electron Microscopy (TEM) imaging of irradiated particles, the effects of irradiation were tracked for increasing fluences [6]. It was seen that satellite particles are formed around the main $\mathrm{Au}$ core (Figure 2, A and B) and neighbouring silica shells fuse together (Figure 2, C and D). These effects will determine the suitable fluences when imaging nanoparticles with $20 \mathrm{keV}$ helium ions. 

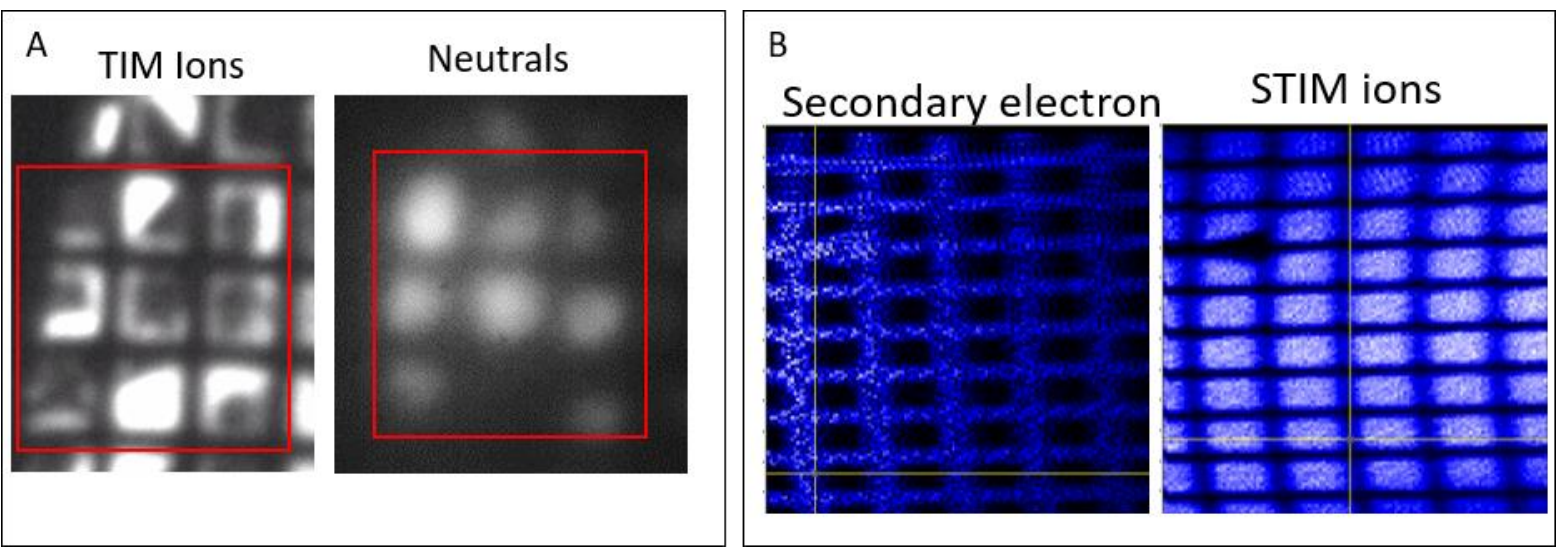

Figure 1. A) TIM images formed with ions and neutrals the sample is 300-mesh lacey carbon grid covered with a single layer graphene membrane, pitch $85 \mu \mathrm{m}(31 \mu \mathrm{m}$ bar, $54 \mu \mathrm{m}$ hole $)$. B) Secondary electron and STIM images recorded concurrently in scanning mode (using MCP/anode plate detection).

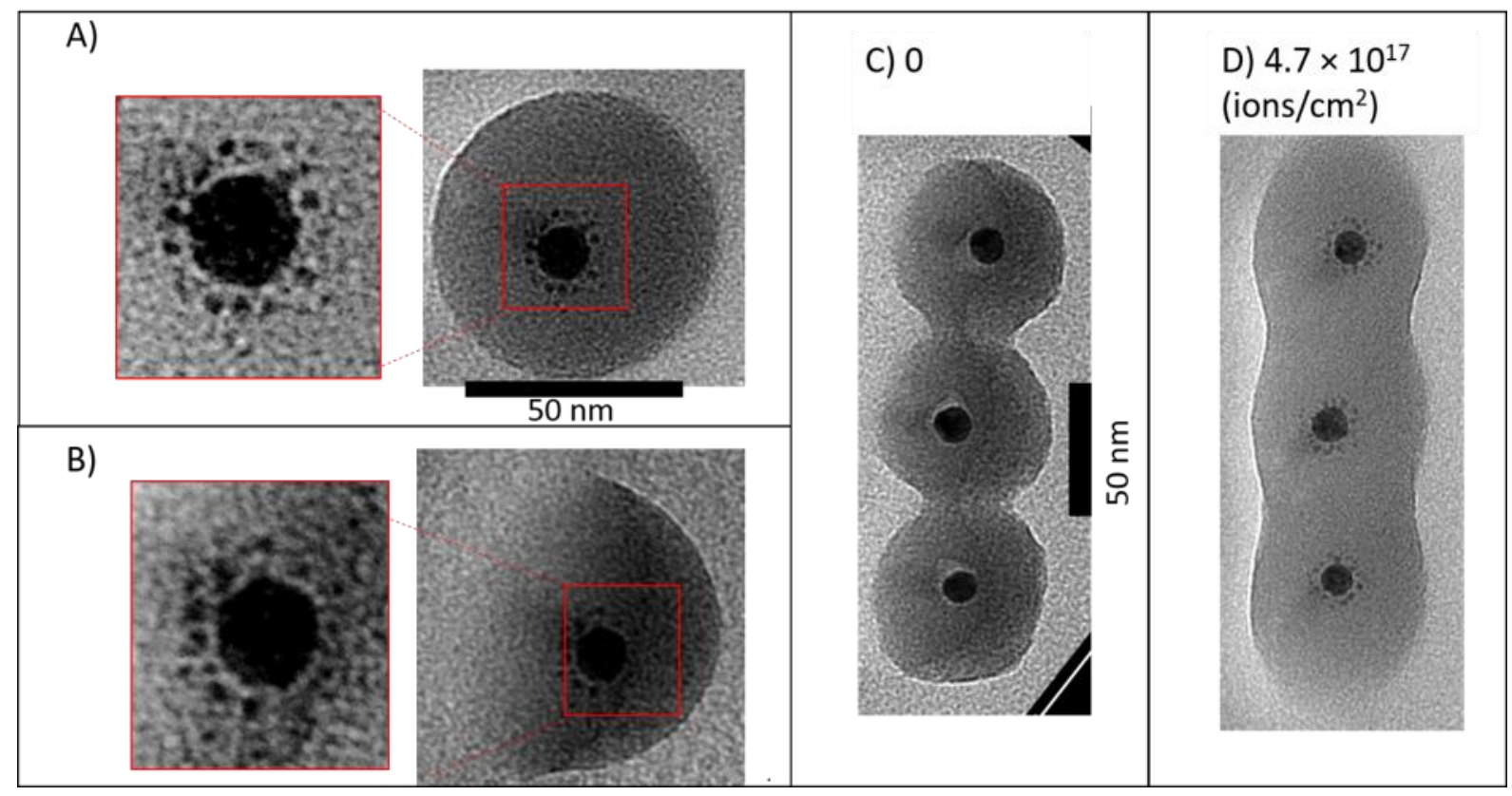

Figure 2. Bright field TEM images at 0 degrees tilt (A) and 60 degrees tilt (B) of a core-shell particle after 4.7x1017 ions/cm2. Bright field TEM images of a collection of core-shell particles before (C) and after (D) $4.7 \times 1017$ ions/cm2 $20 \mathrm{keV}$ helium ion irradiation.

\section{References}

[1] M. Mousley et al., "Stationary beam full-field transmission helium ion microscopy using sub-50 keV He+: Projected images and intensity patterns," Beilstein J. Nanotechnol., vol. 10, pp. 1648-1657, Aug. 2019, doi: 10.3762/bjnano.10.160. [Online]. Available: https://www.beilsteinjournals.org/bjnano/articles/10/160

[2] K. L. Kavanagh C. Herrmann and J. A. Notte, "Camera for transmission He + ion microscopy," J. Vac. Sci. Technol. B, Nanotechnol. Microelectron. Mater. Process. Meas. Phenom., vol. 35, no. 6, p. 
06G902, 2017, doi: 10.1116/1.4991898. [Online]. Available: http://avs.scitation.org/doi/10.1116/1.4991898

[3] T. Wirtz O. De Castro J.-N. Audinot and P. Philipp, "Imaging and Analytics on the Helium Ion Microscope," Аnпи. Rev. Anal. Chem., vol. 12, no. 1, 2019, doi: 10.1146/annurev-anchem-061318115457.

[4] E. Serralta et al., "Scanning transmission imaging in the helium ion microscope using a microchannel plate with a delay line detector," Beilstein J. Nanotechnol., vol. 11, pp. 1854-1864, 2020, doi: 10.3762/BJNANO.11.167.

[5] N. Klingner R. Heller G. Hlawacek S. Facsko and J. von Borany, "Time-of-flight secondary ion mass spectrometry in the helium ion microscope," Ultramicroscopy, vol. 198, no. March 2019, pp. 10-17, 2019, doi: 10.1016/j.ultramic.2018.12.014. [Online]. Available: https://doi.org/10.1016/j.ultramic.2018.12.014 [6] M. Mousley et al., "Structural and chemical evolution of Au-silica core-shell nanoparticles during $20 \mathrm{keV}$ helium ion irradiation: a comparison between experiment and simulation," Sci. Rep., vol. 10, no. 1, pp. 1-13, 2020, doi: 10.1038/s41598-020-68955-7. [Online]. Available: https://doi.org/10.1038/s41598-020-68955-7 